\title{
Sorting on Periodic Surfaces
}

\author{
A. M. Lacasta, ${ }^{1}$ J. M. Sancho, ${ }^{2}$ A. H. Romero, ${ }^{3}$ and Katja Lindenberg ${ }^{4}$ \\ ${ }^{1}$ Departament de Física Aplicada, Universitat Politècnica de Catalunya, Avinguda Doctor Marañon 44, E-08028 Barcelona, Spain \\ ${ }^{2}$ Departament d'Estructura i Constituents de la Matèria, Facultat de Física, Universitat de Barcelona, Diagonal 647, \\ E-08028 Barcelona, Spain \\ ${ }^{3}$ Cinvestav-Queretaro, Libramiento Norponiente No 2000, Real de Juriquilla, 76230 Queretaro, Qro, Mexico \\ ${ }^{4}$ Department of Chemistry and Biochemistry 0340 and Institute for Nonlinear Science, University of California, \\ San Diego, La Jolla, California 92093-0340, USA \\ (Received 22 February 2005; published 29 April 2005)
}

\begin{abstract}
Particles moving on crystalline surfaces and driven by external forces or flow fields can acquire velocities along directions that deviate from that of the external force. This effect depends upon the characteristics of the particles, most notably particle size or particle index of refraction, and can therefore be (and has been) used to sort different particles. We introduce a simple model for particles subject to thermal fluctuations and moving in appropriate potential landscapes. Numerical results are compared to recent experiments on landscapes produced with holographic optical tweezers and microfabricated technology. Our approach clarifies the relevance of different parameters, the direction and magnitude of the external force, particle size, and temperature.
\end{abstract}

DOI: 10.1103/PhysRevLett.94.160601

The rich phenomenology of the transport of particles on crystalline surfaces is attracting enormous interest for its technological relevance. Here we focus on sorting phenomena when mesoscopic particles in a thermal bath and in the presence of an external force or flow field move on a surface modeled as a periodic two-dimensional potential. This work is motivated by recent experiments either in colloidal transport on a two-dimensional periodic potential landscape generated with an array of optical tweezers [13], or DNA separation using microfabricated technology [4]. Sorting is based on the fact that the direction of motion of the particles deviates from that of the external force or flow field, and that the angle of the deviation depends on particle characteristics, most notably particle size. Sorting of a mixture of particles is then possible through collection at different angles.

It is possible to create a landscape with multiple wells (traps) in any configuration using diffractive optical elements to build holographic tweezers [5]. For a particular distribution of wells, the hologram is generated by a computer and etched on a glass plate using standard lithographic procedures. These technologies allow not only the control of the density and specific arrangement (e.g., periodic or random) of wells, but also of the individual well parameters such as width and depth. Thus one can study transport of mesoscopic particles in a highly controlled scenario with the possibility of tracking individual particles. These ideas have been applied to the study of transport and size-dependent sorting of colloidal spheres [1-3]. An alternative technique involves the creation of periodic obstacles on a surface by microfabricated technology. DNA fragments or disperse microspheres in a fluid are drifted by a forced laminar flow [4]. The obstacles cause the particle flow to deviate from the direction of the laminar flow by an angle that depends on particle character-
PACS numbers: 05.60.Cd, 05.40.-a, 66.30.-h, 82.70.Dd

istics such as size or index of refraction. Sorting of particles can thus again be accomplished.

The efficiency of these sorting devices depends on the parameters of the surface potentials and of the particles, the particle interactions with the surface, the external forces, and the temperature. Thermal fluctuations play a much larger role for wells than for obstacles because particles move from one well to another by emerging from one as a result of thermal fluctuations and moving over intermediate plateaus until trapped by another [1,2]. In the case of obstacles, the motion is fairly impervious to thermal fluctuations and is determined mainly by the details of the obstacle course, but now the obstacle configuration (e.g., symmetric or tilted, square lattice or lattice of a different geometry) plays a much more important role [4]. While these effects have been ascertained experimentally for particular cases, there is little available theory to explore the effects of different parameters and different configurations systematically so as to determine the optimal conditions for sorting. Ours is an initial effort in that direction.

We model the above scenarios as an ensemble of classical particles obeying a standard Langevin equation moving in two-dimensional periodic potentials [6] and subjected to an external force $\mathbf{f}$ of modulus $f_{0}$ and direction described by the angle $\theta$,

$$
\mathbf{f}(t)=f_{0}[\cos \theta \mathbf{i}+\sin \theta \mathbf{j}] .
$$

Here we present numerical results for a surface containing wells (traps). Further details and results not presented here, as well as results for surfaces with obstacles, will be discussed in another publication [7].

Broadly speaking, in the system with wells the experiments show that (i) the component of the particle velocity perpendicular to the applied force is sensitively dependent on the direction of the force, (ii) the dependence of the 
direction of the velocity on $\theta$ exhibits a series of plateaus that have been called "kinetically locked-in states" [1], (iii) "randomization" plays a role in the $\theta$ dependence of these plateaus ("statistically locked-in transport" [2]), and (iv) there is a dependence on particle size that leads to the capability of sorting. Our approach will reproduce and provide a physical clarification for all of these features. Similarly, we reproduce and can explain the behaviors observed with obstacles.

Our model consists of noninteracting (dilute) particles moving on a two-dimensional periodic potential $V$ of characteristic length scale $\lambda$. The particles are subjected to the external force (1) and also to thermal noise and the associated dissipation. The equations of motion for a particle of mass $m$ are

$$
\begin{aligned}
& m \ddot{x}=-\frac{\partial}{\partial x} V\left(\frac{x}{\lambda}, \frac{y}{\lambda}\right)-\mu \dot{x}+f_{x}+\xi_{x}(t) \\
& m \ddot{y}=-\frac{\partial}{\partial y} V\left(\frac{x}{\lambda}, \frac{y}{\lambda}\right)-\mu \dot{y}+f_{y}+\xi_{y}(t),
\end{aligned}
$$

where an overdot denotes a derivative with respect to $t$. The parameter $\mu$ is the phenomenological coefficient of friction, and the $\xi_{i}(t)$ are mutually uncorrelated white (thermal) noises that obey the fluctuation-dissipation relation $\left\langle\xi_{i}(t) \xi_{j}\left(t^{\prime}\right)\right\rangle=2 \mu k_{B} T \delta_{i j} \delta\left(t-t^{\prime}\right)$. The potential is chosen to exhibit the main features of the potentials in the experimental studies:

$$
V\left(\frac{x}{\lambda}, \frac{y}{\lambda}\right)=\frac{V_{0}}{1+e^{-g(x, y)}},
$$

where $g(x, y)$ is the periodic two-dimensional function $g(x, y)=A[\cos (2 \pi x / \lambda)+\cos (2 \pi y / \lambda)-2 B]$. The parameter $V_{0}$ controls the depth or height of wells or obstacles, $A$ controls the steepness of these features, and $B$ determines the relative size of a well or obstacle with respect to the spatial period $\lambda$. Figure 1 shows the potential for typical parameters used in our simulations. Note that

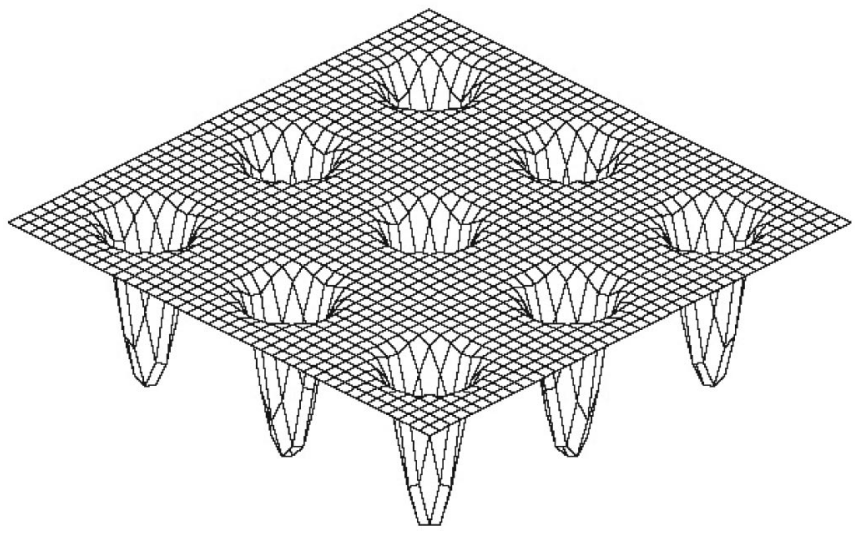

FIG. 1. A finite portion of the much larger two-dimensional periodic potential Eq. (3) for the case of wells with $A=5$ and $B=0.8$. (as in [1]) there are wells but no barriers; the terrain between the traps is essentially flat. The parameter $B$ can be associated with the size of the diffusing particle; larger values of $B$ lead to shallower and narrower wells and are therefore associated with larger particles. The same figure upside down mimics the system with obstacles as used in [4].

Equations (2) can be written in terms of the scaled dimensionless variables $r_{x}=x / \lambda, r_{y}=y / \lambda$, and $\tau=$ $\sqrt{V_{0}} t / \sqrt{m} \lambda$. This scaling serves to stress that for a fixed potential there are only three independent parameters in this model: the scaled temperature $\mathcal{T}$, the scaled dissipation $\gamma$, and the scaled magnitude $F_{0}$ of the external force, given, respectively, by

$$
\mathcal{T}=k_{B} T / V_{0} ; \quad \gamma=\mu \lambda / \sqrt{m V_{0}} ; \quad F_{0}=f_{0} \lambda / V_{0} .
$$

Note that since these potentials can be generated analytically and the equations of motion are continuous, the system is infinite and it is not necessary to specify any particular boundary conditions. Here we do not explore variations in $\gamma$ and $A$, which we fix at $\gamma=20$ and $A=5$.

We wish to explore the direction and magnitude of the velocity of the particles averaged over the thermal fluctuations, $\langle\mathbf{v}\rangle$, compared to those of the external force, as a function of the parameters $B$ (as a measure of particle size), $F_{0}$, and $\mathcal{T}$. From our numerical simulations we evaluate the Cartesian components of the velocity, $\left\langle v_{i}\right\rangle=$ $\lim _{\tau \rightarrow \infty}\left\langle r_{i}(t)\right\rangle / \tau(i=x, y)$, and construct the velocity components parallel and perpendicular to the external force $\mathbf{f}$, $\left\langle v_{\|}\right\rangle$, and $\left\langle v_{\perp}\right\rangle$,

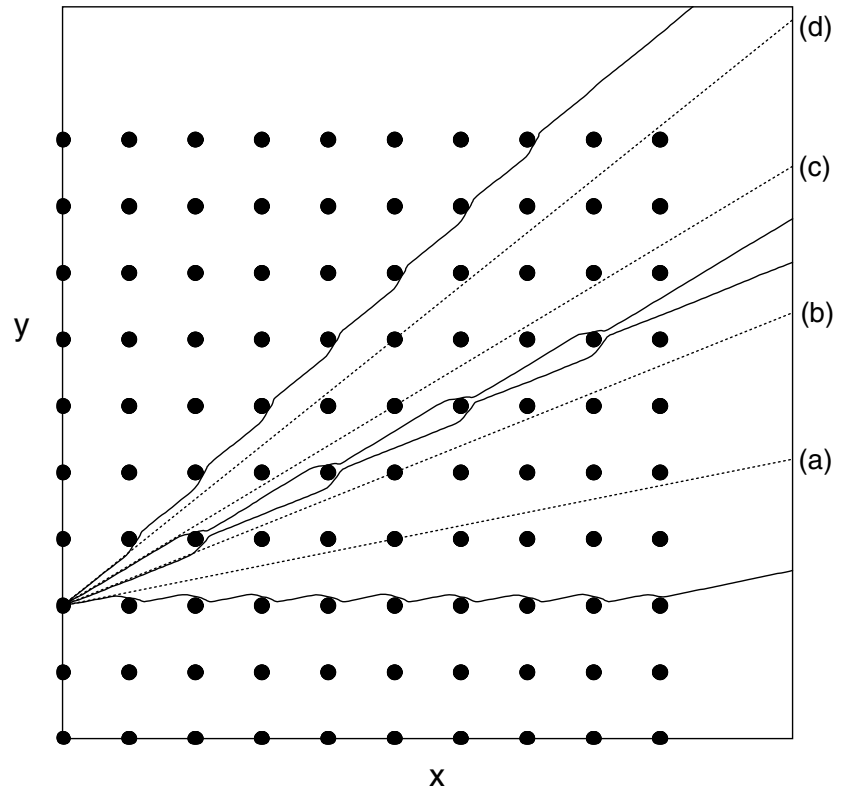

FIG. 2. Trajectories for a force applied at different angles $\theta$. These angles are represented by dotted lines, and correspond to $\tan \theta=0.2$ (a), 0.4 (b), 0.6 (c), and 0.8 (d). Other parameters are: $A=5, B=0.7, \gamma=20, F_{0}=8$, and $\mathcal{T}=10^{-4}$. 


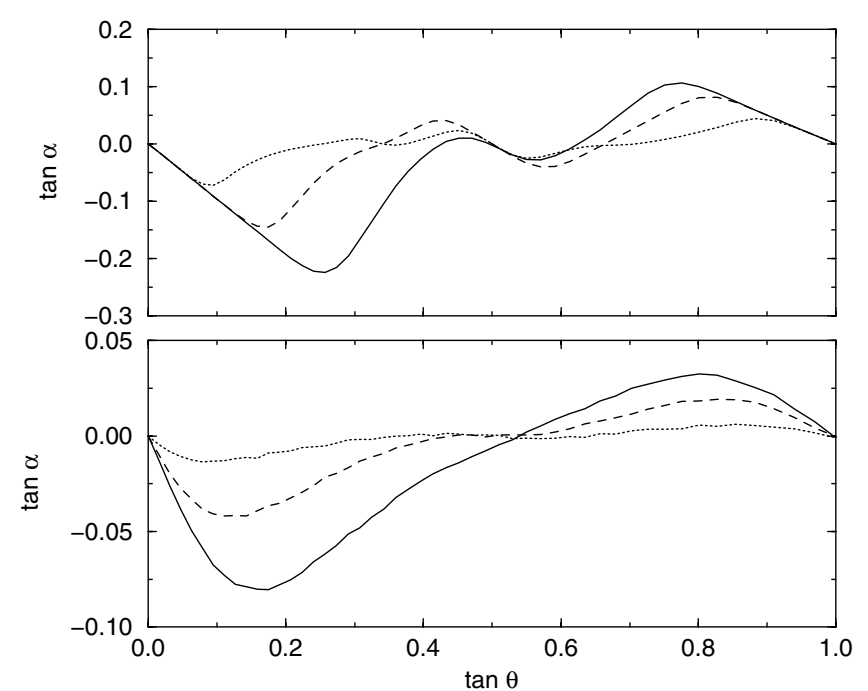

FIG. 3. Deflection angle vs field direction for $F_{0}=8$ and different values of the potential parameter $B$ (increasing $B$ is associated with increasing particle size) for two temperatures. Upper panel $\mathcal{T}=0.01$; lower panel $\mathcal{T}=0.1$. Data correspond to $B_{1}=0.5$ (solid lines), $B_{2}=0.7$ (dashed lines), and $B_{3}=0.9$ (dotted lines).

$$
\begin{gathered}
\left\langle\boldsymbol{v}_{\|}\right\rangle=\left\langle\boldsymbol{v}_{x}\right\rangle \cos \theta+\left\langle\boldsymbol{v}_{y}\right\rangle \sin \theta, \\
\left\langle\boldsymbol{v}_{\perp}\right\rangle=-\left\langle\boldsymbol{v}_{x}\right\rangle \sin \theta+\left\langle\boldsymbol{v}_{y}\right\rangle \cos \theta .
\end{gathered}
$$

The deviation of $\langle\mathbf{v}\rangle$ from $\mathbf{f}$ can be analyzed either through the absolute velocity angle $\Psi$ or the deflection angle $\alpha$ (note the relation $\Psi=\theta+\alpha$ ),

$$
\tan \Psi \equiv \frac{\left\langle v_{y}\right\rangle}{\left\langle v_{x}\right\rangle}, \quad \tan \alpha \equiv \frac{\left\langle v_{\perp}\right\rangle}{\left\langle v_{\|\rangle}\right.} .
$$

In Fig. 2 we present a number of typical trajectories corresponding to the potential (3) with parameter values that lead to periodic traps connected by flat regions (see figure caption for parameter values). The temperature in these realizations is very low. On the basis of such trajectories we can obtain the desired dependences, which we present in a number of figures and discuss below. We note that the source of the angle separation between $\langle\mathbf{v}\rangle$ and $\mathbf{f}$ is already clearly apparent in Fig. 2.

The dependence of the absolute velocity angle $\Psi$ and of the deflection angle $\alpha$ on $\theta$ is the source of the sorting capability, and is clearly seen in Figs. 3-5 as a function of different parameters. Figure 3 makes it clear that at a low temperature (upper panel) there is a direction of the force that leads to maximal deviation of $\alpha$ from zero for a given particle size, while other force directions also lead to pronounced but smaller deviations. It is also clear that the optimal angle for sorting depends on the sizes of the particles to be sorted. For instance, in the case shown in the figure, a mixture of $B_{1}$ and $B_{2}$ particles is most effectively separated if the force is at an angle $\tan \theta \sim 0.25$ from the $[1,0]$ direction, but a mixture of $B_{2}$ and $B_{3}$ particles is
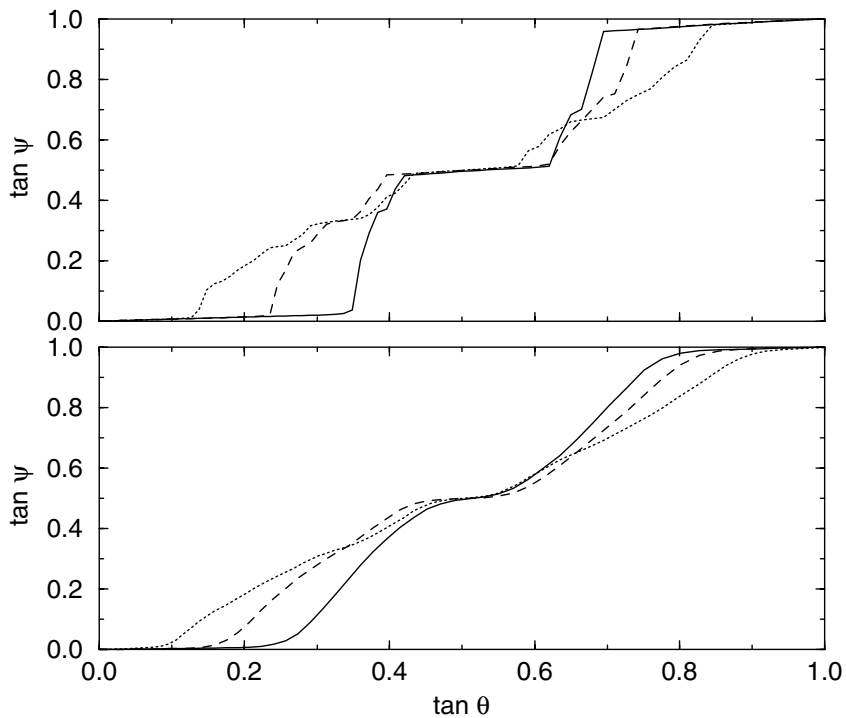

FIG. 4. Plateaus in the absolute velocity angle for $F_{0}=8$ and different values of the parameter $B$ for two temperatures. Upper panel $\mathcal{T}=10^{-4}$; lower panel $\mathcal{T}=10^{-2}$. Data correspond to $B=0.5$ (solid lines), 0.7 (dashed lines), and 0.9 (dotted lines).

better sorted using an angle $\tan \theta \sim 0.15$. With increasing temperature the trajectories become more erratic as thermal fluctuations agitate the particles away from the direction of the force and of the nearest wells. As seen in the lower panel of the figure, the deflection angle is now smaller than before, but there is still an angle $\theta$ for maximum deviation from $\alpha=0$ that varies with particle size. The temperature increase has washed out all but one minimum and one maximum in the deflection angle as a function of $\theta$. This figure compares favorably, not only

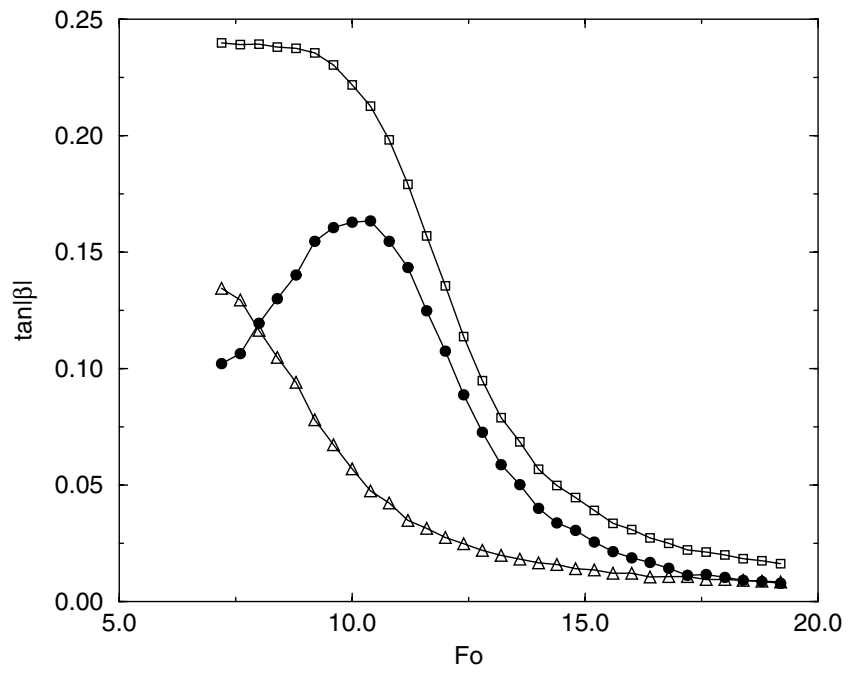

FIG. 5. Deflection angles $\alpha_{i}$ for different particles $B_{i}$ as a function of $F_{0}$ for a fixed force direction $\tan \theta=0.25$ and $\mathcal{T}=$ 0.01. Squares: $B_{1}=0.5, \beta \equiv \alpha_{1}$. Triangles: $B_{2}=0.7, \beta \equiv \alpha_{2}$. Circles: difference between deflection angles of the two types of particles, $\beta \equiv \alpha_{2}-\alpha_{1}$. 
qualitatively but even quantitatively with Fig. 4 of Ref. [1]. At sufficiently high temperature the effect disappears as the wells become irrelevant. We find (not shown here) that the approach of $\alpha$ to zero at a given $\theta$ is monotonic with increasing particle size.

The terrace phenomenon in the dependence of the absolute velocity angle on the direction of the force has been observed and discussed extensively [1,2] (see Fig. 5 in [1] and Figs. 2 and 3 in [2]) and is seen here as well in Fig. 4. The plateaus have been called "kinetically locked-in states" and can be understood on the basis of the trajectories in Fig. 2. All the trajectories included in a small cone targeting a particular well will emerge out of this well within an identical cone [see the cone between lines (b) and (c) in the figure]. The terraces are clearly pronounced at low temperatures, the main ones occurring at $\Psi=0$ and $\Psi=\pi / 4$, with the next most persistent around $\tan \Psi=$ 0.5. As the temperature increases, the particle agitation disturbs and washes out the smaller cones (this corresponds to the notion of "statistically locked-in transport" in Ref. [2]), leading to the eventual disappearance of the plateaus (see the lower panel in Fig. 4). As might be expected from the above explanation of the source of the plateau effect, the terraces are more pronounced for smaller particles.

Finally, consider the dependence of the deflection angle on the magnitude $F_{0}$ of the external force. This dependence is shown in Fig. 5, where, for a given force direction, we show the deflection angle of two types of particles as a function of $F_{0}$. We also show the dependence of the difference of deflection angles of the two types of particles on $F_{0}$. The main point here is that sorting is more effective at smaller forces, a reasonable result since a sufficiently large force simply pulls the particles along regardless of the presence of traps. This effect has been seen experimentally; see Fig. 3 in Ref. [3].

We have carried out an analogous detailed study of particles moving on a potential surface of periodic obstacles separated by flat regions [4]. Although experiments have shown that greater sorting efficiencies can be achieved by tilting the objects and, even more, by placing them in configurations other than a square lattice, even the simple square lattice (the upside down configuration of Fig. 1) leads to sorting. The particles hit the obstacles and deviate from the direction of the force as determined by reflection laws. Thermal fluctuations play a much less important role in this case. Although the trajectories of the particles here are different in detail than in the case of the traps, the phenomenology is very similar. The ingredients of the sorting phenomenon and the plateau structure are again present in this case. A detailed study will appear elsewhere [7].

In conclusion, we have formulated a simple Langevin model that explains all the features that have been seen in experiments on colloidal transport over surfaces that con- tain periodically located wells (traps) (generated with an array of optical tweezers) and DNA separation with periodically located obstacles (constructed using microfabricated technology) in the presence of an externally imposed force or flow field. The behaviors of greatest practical interest include the fact that the direction of motion of the particles deviates from the direction of the force by an amount that can be optimized by adjusting the direction of the force, and that this deviation is particle size dependent (or dependent on some other characteristic of the particle such as its index of refraction). Therefore, particles can be sorted as they move across such surfaces. A second aspect of the behavior that has been highlighted is the appearance of plateaus in the dependence of the average direction of the particle motion on the direction of the force. Our model captures both of these behaviors, as well as observed and predicted dependences on temperature and on the magnitude of the external force. We stress that the potentials and thermal fluctuations are completely symmetric, so that the observed phenomena are not associated with any kind of ratchet effect. This study again confirms the utility and experimental relevance of the classical Langevin dynamical scenario in the study of diffusion of mesoscopic particles on surfaces.

This work was supported by the MCyT (Spain) under project BFM2003-07850, by the Engineering Research Program of the Office of Basic Energy Sciences at the U. S. Department of Energy under Grant No. DE-FG0204ER46179, and by a grant from the University of California Institute for México and the United States (UC MEXUS) and the Consejo Nacional de Ciencia y Tecnología de México (CoNaCyT). A. H. R. acknowledges support from CONACyT-Mexico through Grant No. J42647-F.

[1] P. T. Korda, M. B. Taylor, and D. G. Grier, Phys. Rev. Lett. 89, 128301 (2002).

[2] A. Gopinathan and D. G. Grier, Phys. Rev. Lett. 92, 130602 (2004).

[3] M.P. MacDonald, G. C. Spalding, and K. Dholakia, Nature (London) 426, 421 (2003).

[4] L. R. Huang, E. C. Cox, R. H. Austin, and J. C. Sturm, Anal. Chem. 75, 6963 (2003); Science 304, 987 (2004).

[5] K. Dholakia, G. Spalding, and M. MacDonald, Phys. World (October), 31 (2002), http://physicsweb.org/articles/world/15/10/8; D. G. Grier, Nature (London) 424, 810 (2003).

[6] J. M. Sancho, A. M. Lacasta, K. Lindenberg, I. M. Sokolov, and A. H. Romero, Phys. Rev. Lett. 92, 250601 (2004); A. M. Lacasta, J. M. Sancho, A. H. Romero, I. M. Sokolov, and K. Lindenberg, Phys. Rev. E 70, 051104 (2004); K. Lindenberg, A. M. Lacasta, J. M. Sancho, and A. H. Romero, New J. Phys. 7, 29 (2005).

[7] A. M. Lacasta, J.M. Sancho, A.H. Romero, and K. Lindenberg (in preparation). 